\title{
Front Matter: Volume 10701
}

, "Front Matter: Volume 10701," Proc. SPIE 10701, Optical and Infrared Interferometry and Imaging VI, 1070101 (29 August 2018); doi:

$10.1117 / 12.2506190$

SPIE Event: SPIE Astronomical Telescopes + Instrumentation, 2018, Austin, Texas, SPIE. United States 


\title{
PROCEEDINGS OF SPIE
}

\section{Optical and Infrared Interferometry and Imaging VI}

\author{
Michelle J. Creech-Eakman \\ Peter G. Tuthill \\ Antoine Mérand \\ Editors
}

11-15 June 2018

Austin, Texas, United States

Sponsored by

SPIE

Cosponsored by

4D Technology (United States) - Andor Technology, Ltd. (United Kingdom) - Astronomical Consultants \& Equipment, Inc. (United States) - Giant Magellan Telescope (Chile) - GPixel, Inc. (China) • Harris Corporation (United States) • Materion Corporation (United States) Optimax Systems, Inc. (United States) - Princeton Infrared Technologies (United States) Symétrie (France) • Teledyne Technologies, Inc. (United States) • Thirty Meter Telescope (United States)

\section{Cooperating Organizations}

European Space Organisation - National Radio Astronomy Observatory (United States) Science \& Technology Facilities Council (United Kingdom) • Canadian Astronomical Society (Canada) • Canadian Space Association ASC (Canada) • Royal Astronomical Society (United Kingdom) - Association of Universities for Research in Astronomy (United States) American Astronomical Society (United States) - Australian Astronomical Observatory (Australia) • European Astronomical Society (Switzerland)

Published by

SPIE

Volume 10701

Part One of Two Parts

Proceedings of SPIE 0277-786X, V. 10701

SPIE is an international society advancing an interdisciplinary approach to the science and application of light.

Optical and Infrared Interferometry and Imaging VI, edited by Michelle J. Creech-Eakman,

Peter G. Tuthill, Antoine Mérand, Proc. of SPIE Vol. 10701, 1070101 · @ 2018 SPIE

CCC code: $0277-786 \mathrm{X} / 18 / \$ 18 \cdot$ doi: $10.1117 / 12.2506190$ 
The papers in this volume were part of the technical conference cited on the cover and title page. Papers were selected and subject to review by the editors and conference program committee. Some conference presentations may not be available for publication. Additional papers and presentation recordings may be available online in the SPIE Digital Library at SPIEDigitalLibrary.org.

The papers reflect the work and thoughts of the authors and are published herein as submitted. The publisher is not responsible for the validity of the information or for any outcomes resulting from reliance thereon.

Please use the following format to cite material from these proceedings:

Author(s), "Title of Paper," in Optical and Infrared Interferometry and Imaging VI, edited by Michelle J. Creech-Eakman, Peter G. Tuthill, Antoine Mérand, Proceedings of SPIE Vol. 10701 (SPIE, Bellingham, WA, 2018) Seven-digit Article CID Number.

ISSN: 0277-786X

ISSN: 1996-756X (electronic)

ISBN: 9781510619555

ISBN: 9781510619562 (electronic)

Published by

SPIE

P.O. Box 10, Bellingham, Washington 98227-0010 USA

Telephone +1 3606763290 (Pacific Time) · Fax +1 3606471445

SPIE.org

Copyright (c) 2018, Society of Photo-Optical Instrumentation Engineers.

Copying of material in this book for internal or personal use, or for the internal or personal use of specific clients, beyond the fair use provisions granted by the U.S. Copyright Law is authorized by SPIE subject to payment of copying fees. The Transactional Reporting Service base fee for this volume is $\$ 18.00$ per article (or portion thereof), which should be paid directly to the Copyright Clearance Center (CCC), 222 Rosewood Drive, Danvers, MA 01923. Payment may also be made electronically through CCC Online at copyright.com. Other copying for republication, resale, advertising or promotion, or any form of systematic or multiple reproduction of any material in this book is prohibited except with permission in writing from the publisher. The CCC fee code is 0277$786 \mathrm{X} / 18 / \$ 18.00$.

Printed in the United States of America.

Publication of record for individual papers is online in the SPIE Digital Library.

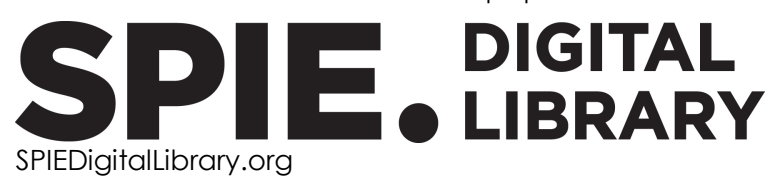

Paper Numbering: Proceedings of SPIE follow an e-First publication model. A unique citation identifier (CID) number is assigned to each article at the time of publication. Utilization of CIDs allows articles to be fully citable as soon as they are published online, and connects the same identifier to all online and print versions of the publication. SPIE uses a seven-digit CID article numbering system structured as follows:

- The first five digits correspond to the SPIE volume number.

- The last two digits indicate publication order within the volume using a Base 36 numbering system employing both numerals and letters. These two-number sets start with 00, 01, 02, 03, 04, 05, 06, 07, 08, 09, OA, OB ... 0Z, followed by 10-1Z, 20-2Z, etc. The CID Number appears on each page of the manuscript. 


\title{
Contents
}

\author{
xi Authors \\ xvii Conference Committee \\ xix Introduction
}

\section{Part One}

CURRENT AND PLANNED FACILITIES I

1070102 Recent technical and scientific highlights from the CHARA array (Invited Paper) [10701-1]

1070103 VLTI status update: three years into the second generation (Invited Paper) [10701-2]

CURRENT AND PLANNED FACILITIES II

1070105 Many interesting things are afoot at the Navy Precision Optical Interferometer (Invited Paper) [10701-4]

1070106 The Magdalena Ridge Observatory interferometer: first light and deployment of the first telescope on the array (Invited Paper) [10701-5]

1070107 The GRAVITY fringe tracker: correlation between optical path residuals and atmospheric parameters [10701-6]

CURRENT AND PLANNED FACILITIES III

1070109 Commissioning MATISSE: first results [10701-8]

$10701 \mathrm{OB} \quad$ An infrared beam combiner for wavelength bootstrapping at the NPOI [10701-10]

POSTER SESSION: CRITICAL SUBSYSTEMS

10701 OC Prototyping FOURIER: the first generation near-infrared science beam combiner at the MROI [10701-60] 
10701 OD The MROI fringe tracking system: camera hardware modifications to integrate the SAPHIRA detector [10701-61]

10701 OF Control of piezoelectric actuator with hysteresis and creep [10701-63]

POSTER SESSION: CURRENT AND PLANNED FACILITIES AND INSTRUMENTS

10701 OG NESSI and 'Alopeke: two new dual-channel speckle imaging instruments [10701-65]

$10701 \mathrm{OH}$ MATISSE: performance in laboratory, results of AIV in Paranal, and first results on sky [10701-66]

$1070101 \quad$ Initial steps toward a new method of atmospheric characterization over long baseline arrays [10701-67]

$107010 \mathrm{~J} \quad$ Towards controlled Fizeau observations with the Large Binocular Telescope [10701-68]

10701 OK Improving GRAVITY towards observations of faint targets [10701-69]

10701 OM Augmented design for a fully automated alignment system at the Magdalena Ridge Observatory Interferometer [10701-71]

10701 ON NAOMI: the adaptive optics for the auxiliary telescopes of VLTI [10701-72]

1070100 Magdalena Ridge Observatory interferometer: UT\#1 site installation, alignment and test [10701-73]

$10701 \mathrm{OP}$ Towards integration of the Unit Telescope for the Magdalena Ridge Observatory interferometer [10701-74]

POSTER SESSION: FUTURE OF INTERFEROMETRY

$107010 Q$ Can telescopes with elongated pupils achieve higher contrast and resolution? [10701-76]

TECHNIQUES I

10701 OS Masking interferometry at 150: old enough to mellow on redundancy? (Invited Paper) [10701-11]

10701 OT Multiplexed holographic aperture masking with liquid-crystal geometric phase masks [10701-12]

10701 OU Hi-5: a potential high-contrast thermal near-infrared imager for the VLTI [10701-13] 
10701 OV GLINT South: a photonic nulling interferometer pathfinder at the Anglo-Australian Telescope for high contrast imaging of substellar companions [10701-14]

\section{TECHNIQUES II}

10701 OW Implementation of an intensity interferometry system on the StarBase observatory [10701-16]

$107010 X \quad$ Stellar photon correlation detection with the Southern Connecticut stellar interferometer [10701-17]

10701 OY Prospects for wireless optical intensity interferometry with the Southern Connecticut stellar interferometer [10701-18]

$10701 \mathrm{OZ}$ The installation and ongoing commissioning of the MATISSE mid-infrared interferometer at the ESO Very Large Telescope Observatory (Invited Paper) [10701-54]

\section{TECHNIQUES III}

$1070110 \quad$ Wide-field speckle techniques for small, urban telescopes [10701-20]

$1070111 \quad$ Image-plane fringe tracker for adaptive-optics assisted long baseline interferometry [1070121]

1070112 The current state of speckle imaging (Invited Paper) [10701-19]

\section{FUTURE OF INTERFEROMETRY I}

1070114 The VLTI roadmap for the next decade (Invited Paper) [10701-23]

1070116 A six-apertures discrete beam combiners for J-band interferometry [10701-25]

\section{FUTURE OF INTERFEROMETRY II}

$1070118 \quad$ Planet formation imager: project update (Invited Paper) [10701-27]

1070119 A three-telescope active integrated optics spectro-interferometric combiner in the L-band for application to high precision interferometry [10701-28]

10701 1B Photonics-based mid-infrared interferometry: the challenges of polychromatic operation and comparative performances [10701-30] 


\section{DATA PROCESSING I}

$107011 \mathrm{C}$ Synergy between radio and optical interferometry: image reconstruction, calibration and data analysis (Invited Paper) [10701-31]

10701 1D Comparing non-redundant masking and filled-aperture kernel phase for exoplanet detection and characterization [10701-32]

10701 IE Photonic mid-infrared nulling for exoplanet detection on a planar chalcogenide platform [10701-33]

\section{SPACE INTERFEROMETRY}

$107011 G \quad$ A dispersive backend design for the 'double-Fourier' interferometer BETTII (Invited Paper) [10701-35]

$10701 \mathrm{lH} \quad$ Characterizing the atmosphere of Proxima b with a space-based mid-infrared nulling interferometer [10701-36]

1070111 Exoplanet science with a space-based mid-infrared nulling interferometer [10701-37]

\section{Part Two}

\section{SPACE INTERFEROMETRY}

10701 1J The TOLIMAN space telescope [10701-38]

$107011 \mathrm{~K}$ The wide-field spatio-spectral interferometer: system overview, data synthesis and analysis [10701-39]

\section{DATA PROCESSING II}

$10701 \mathrm{lL} \quad$ Protoplanetary environments at the astronomical unit scale: the contribution of long baseline interferometry (Invited Paper) [10701-40]

10701 1M A two-band approach to $\mathrm{n} \lambda$ phase error corrections with LBTI's PHASECam [10701-41]

$10701 \mathrm{lN} \quad$ Coherent integration: theory and practice [10701-43]

1070110 Panel discussion: next steps for OIFITS (Invited Paper) [10701-44]

10701 1P Correction of differential chromatic dispersion in GRAVITY [10701-79] 
TECHNOLOGIES I

10701 1Q Astrophotonic interferometry: coherently moulding the flow of starlight (Invited Paper) [10701-45]

10701 IR Photonics-based mid-infrared interferometry: 4-year results of the ALSI project and future prospects [10701-46]

10701 is Visible and near-infrared multilayer arrayed waveguide gratings: a novel approach for spectro-interferometry [10701-47]

10701 IT Staring at stars makes you see spots: verifying images of red supergiant stars [10701-48]

IMAGING BEAUTY CONTEST WINNERS

$107011 \mathrm{U} \quad$ Optical interferometry image reconstruction contest VIII [10701-103]

\section{TECHNOLOGIES II}

$10701 \mathrm{lV}$ The success of extragalactic infrared interferometry: from what we have learned to what to expect (Invited Paper) [10701-49]

10701 IW Improving angular resolution of telescopes through probabilistic single-photon amplification? (Invited Paper) [10701-50]

$107011 \mathrm{X}$ Fiber-based infrared heterodyne technology for the PFI: on the possibility of breaking the noise temperature quantum limit with cross-correlation [10701-51]

$107011 Y \quad$ Learnings from the use of fiber optics in GRAVITY [10701-52]

\section{CRITICAL SUBSYSTEMS}

1070120 SPICA: a new 6T visible beam combiner for CHARA: science, design and interfaces (Invited Paper) [10701-55]

1070121 Intensity interferometry revival on the Côte d'Azur (Invited Paper) [10701-15]

\section{CRITICAL SUBSYSTEMS II}

1070122 MYSTIC: Michigan Young STar Imager at CHARA [10701-56] 
1070123 The MIRC-X 6-telescope imager: key science drivers, instrument design and operation [10701-57]

1070124 MIRC-X/CHARA: sensitivity improvements with an ultra-low noise SAPHIRA detector [10701-58]

1070126 More telescopes than photons: beam multiplexing in imaging interferometers [10701-64]

POSTER SESSION: OBSERVING TECHNIQUES

1070127 Simulation and experimental study on the detection of binary stars with non-redundant aperture masking [10701-78]

1070128 Simultaneous ground- and space-based observations in the JWST era [10701-80]

POSTER SESSION: DATA PROCESSING, ANALYSIS, ACCESS, AND DISCOVERY

1070129 Image enhancement for the observation of the solar corona [10701-81]

$107012 \mathrm{~A}$ Two-color speckle imaging of $\mathbf{M}$-dwarfs with the Discovery Channel telescope [10701-82]

$107012 \mathrm{~B} \quad$ SHARK-NIR coronagraphic simulations: performance dependence on the Strehl ratio [10701-83]

$107012 \mathrm{D}$ The fundamentals: angular diameter measurements of zero-crossing stars from the NPOI [10701-85]

$107012 \mathrm{E} \quad$ Speckle imaging of KOI binary stars with the WIYN telescope [10701-86]

$107012 F \quad$ Aperture synthesis imaging of colored GEO objects [10701-87]

$107012 \mathrm{G}$ Common spatial pattern filtering for detection of circumstellar discs [10701-88]

1070121 Detecting exoplanets and characterizing their properties with fringe nulling [10701-90]

POSTER SESSION: TECHNOLOGIES

$107012 \mathrm{~L}$ Fiber-based infrared heterodyne technology for the PFI: development of a prototype test system [10701-94]

$107012 \mathrm{M} \quad$ Heterodyne versus direct detection: it is not over [10701-95]

1070120 Beam combination schemes and technologies for the Planet Formation Imager [10701-97] 
$107012 P \quad$ A large mosaic echelle grating for ESPRESSO spectrograph [10701-98]

$107012 Q$ SoC-based real-time controller for piezo-positioning using Matlab/Simulink [10701-99]

$107012 R \quad$ Very accurate cryogenic mechanisms for CRIRES+ [10701-100]

$107012 T \quad$ Time tagging individual photons with a low-cost FPGA based time to digital converter [10701-102]

$107012 \mathrm{U} \quad$ Speckle imaging through a coherent fiber bundle [10701-105]

$107012 \mathrm{~V}$ First results on an electro-optic visible multi-telescope beam combiner for next generation FIRST/SUBARU instruments [10701-104] 
Proc. of SPIE Vol. 10701 1070101-10

Downloaded From: https://www.spiedigitallibrary.org/conference-proceedings-of-spie on 26 Apr 2023 Terms of Use: https://www.spiedigitallibrary.org/terms-of-use 


\section{Authors}

Numbers in the index correspond to the last two digits of the seven-digit citation identifier (CID) article numbering system used in Proceedings of SPIE. The first five digits reflect the volume number. Base 36 numbering is employed for the last two digits and indicates the order of articles within the volume. Numbers start with 00, 01, 02, 03, 04, 05, 06, 07, 08, 09, OA, OB...0Z, followed by 10-1Z, 20-2Z, etc.

\author{
Abad, José Antonio, 03 \\ Absil, Olivier, OU, 1H, 11 \\ Abuter, Roberto, 03, 07 \\ Adler, $\mathrm{T}$., $\mathrm{OH}$ \\ Agapito, G., 2B \\ Agocs, T., OZ \\ Alberdid, Antxon, $1 \mathrm{U}$ \\ Aller-Carpentier, Emmanuel, 03, ON \\ Allouche, Fatmé, 09, $\mathrm{OH}, \mathrm{OZ}$ \\ Alonso, Jaime, 03, ON \\ Alonso-Herrero, Almudena, 18 \\ Amorim, António, 07, OK, IP, IY \\ Anderson, Matthew D., 02 \\ Andolfato, Luigi, 03, ON \\ Anglada Escude, Guillem, 2R \\ Antonelli, P., OH, $\mathrm{OZ}$ \\ Anugu, Narsireddy, 22, 23, 24 \\ Armstrong, J. Thomas, 05, OB, 2D, 21 \\ Arriola, Alexander, OV, 1B, 1R, 1S \\ Augereau, J.-C., $\mathrm{OZ}$ \\ Bailet, Christophe, $\mathrm{OH}, 0 \mathrm{Z}, 20$ \\ Baines, Ellyn K., 05, OB, 2D, 21 \\ Baptista, Brian J., 2E \\ Barbier, D., 2V \\ Baron, Fabien R., 18, 1T, 2D \\ Barriga, Pablo José, 03 \\ Bastin, Christian, 00 \\ Bayo, Amelia, 18 \\ Becerra, A., 2L \\ Beckmann, U., $\mathrm{OH}, \mathrm{OZ}$ \\ Beltran, Juan, $03, \mathrm{OH}$ \\ Bendek, Eduardo, $1 \mathrm{~J}$ \\ Benech, P., 19 \\ Benson, James A., 05, 2D \\ Berger, Jean-Philippe, 03, 0N, 0U, 18, 19, 1L \\ Bergomi, M., 2B \\ Berio, Philippe, 09, OH, 0Z, 20 \\ Besser, Felipe E., 1X, 2L, 2M \\ Bettonvil, F., $\mathrm{OH}, \mathrm{OZ}$ \\ Bevilacqua, R. G., 05 \\ Biondi, F., 2B \\ Blasi, Robert, 06, OM, OP \\ Blind, Nicolas, $1 Y$ \\ Bolcar, Matthew R., $1 \mathrm{~K}$ \\ Bonsor, Amy, 18 \\ Bourget, Pierre, $03,0 \mathrm{H}, 0 \mathrm{~N}$ \\ Boyajian, Tabetha, 18 \\ Brandner, Wolfgang, 07, OK, IP, IY \\ Brast, Roland, 03
}

Bresson, Yves, $\mathrm{OH}, 0 \mathrm{Z}, 20$

Bristow, Paul, 03, OH, 0Z, 2R

Buron, Alexander, $1 \mathrm{Y}$

Buscher, David F., 06, OC, OD, OM, 26, 2F

Buschmann, T., 05

Cadiz, M. D., 2 L

Caniguante, Luis, 03

Carlotti, A., 2B

Carolo, E., 2B

Carrasco, J., 2L

Cassagnettes, C., $2 \mathrm{~V}$

Cassaing, Frédéric, 20

Chabé, Julien, 21

Chelli, Alain, 09, $0 \mathrm{H}$

Chiavassa, Andrea, 18

Chinellato, S., 2B

Ciardi, David, 18

Clark, Catherine, 2A

Clark, J. H., 05

Clarke, Orville, OW

Clausse, Jean-Michel, $0 \mathrm{H}, 20$

Conrad, Al, 28

Conzelmann, Ralf, 03

Corrielli, G., 16

Cortes, Angela, 03

Courde, Clément, 21

Courjal, N., 19, 2V

Creech-Eakman, Michelle, 06, OD, OI, OP, 18, 2F

Cruzalèbes, Pierre, 09, $\mathrm{OH}, \mathrm{OZ}$

Cvetojevic, Nick, OV, 2V

Dahl, Chuck, 06, OP

Danchi, W. C., OU

Darré, Pascaline, 03, 0N

Davidson, James W., Jr., 2E, $2 \mathrm{U}$

Davies, Claire L., 23, 24

Defrère, Denis, OU, 11, 18, 1H, 11, 1M

DeGroff, W. T., 05

Dejonghe, Julien, 20

Dekker, Hans, 2P

Del Valle, Diego, 03

Delbo, M., OZ

Delboulbé, Alain, 03, 0N

Delplancke-Ströbele, Françoise, 03

Dembet, Roderick, 03, 07, 0N, IP

De Pascale, M., 2B

Derie, Frédéric, 03

de Wit, Willem-Jan, 03, 18

Dhabal, Arnab, $1 G$

Diaz, J., 2L 
Diener, Romina, 1B, 1R, 20

Dima, M., 2B

Doelman, D. S., OT

Dominik, C., $\mathrm{OZ}$

Dong, Ruobing, 18

Donoso, Reinaldo, 03

Dooley, J. F., 06, 0l

D'Orazi, V., 2B

Dorn, Reinhold J., 2R

Douglass, G., $1 \mathrm{~S}$

Downey, E., $1 \mathrm{M}$

Dubravec, Veronika, 24

Duchêne, Gaspard, 18

Duhoux, Philippe, 03

Dupuy, Christophe, 03, 0N

Egner, Sebastien, 03, 0N

Eisenhaver, Frank, 07, 0K, 1P, 1Y

Eldswick, E., $\mathrm{OH}, \mathrm{OZ}$

Ennis, Jacob, 22, 23, 24

Ertel, Steve, 0J, OU, 1M

Escuti, M. J., OT

Espaillat, Catherine, 18

Espinoza, K. R., 2L

Etscorn, Dylan, 06, OP

Everett, Mark E., 2E

Fantei-Caujolle, $\mathrm{Y}_{.,}, 09, \mathrm{OH}, \mathrm{OZ}$

Farinato, J., 2B

Farrington, Christopher D., 02

Farris, Allen, 06, OM, OP

Fedou, P., 07

Fisher, M., 06

Fixsen, Dale, $1 \mathrm{G}$

Flebus, Carlo, 00

Folcher, Jean-Pierre, $\mathrm{OF}, 2 \mathrm{Q}$

Follert, Roman, 2R

Förster, Andreas, 03

Fouché, Mathilde, 21

Frahm, Robert, 03

Fuenteseca, Eloy, 03

Gallenne, Alexandre, $\mathrm{OH}, \mathrm{OU}, 18$

Gandhi, Poshak, 18

Gao, Feng, OK

Garcia Munoz, A., $1 \mathrm{H}$

Garcia, E., 06

Gardillou, F., 2V

Gaytan, Daniel, 03

Genzel, Reinhard, OK, IY

Gies, Douglas R., 02

Gillessen, Stefan, OK, 1 Y

Gino, C., 06

Glauser, Adrian M., 11

Glindemann, Andreas, 03, 09, OH, $\mathrm{OZ}$

Godolt, M., $1 \mathrm{H}$

Golden, Steven, 02

Gonté, Frédéric, 03, 0N

Gonzales, Jaime, 03

Gonzalez, Jean-Francois, 18

Granucci, Nicole M., 10

Greggio, D., 2B

Grenfell, J. L., 1H
Gretzinger, Thomas, OV

Gross, Simon, OV, 1B, 1R, 1S

Guerin, William, 21

Guerlet, Thibaut, 03, $\mathrm{OH}$

Guieu, Sylvain, 03, 0N

Guisard, Stéphane, 03

Guitton, F., OH

Gulinatti, Angelo, OY

Gutierrez, Pablo, 03, 0N

Guyon, Olivier, $1 \mathrm{~J}$

Guyot, C., 2V

Hagino, M., 29

Haguenaver, Pierre, 03, 0N

Hahne, Frederick W., 2A

Haidar, Mariam, 24

Haimerl, Andreas, 03

Hall, J. C., 05

Haniff, Christopher, 06, OD, 18, 2F

Hans, Oliver, IY

Hatzes, Artie, 2R

Haubois, Xavier, 03, 0H, 10, $1 \mathrm{P}$

Hauden, J., 2V

Haug, Marcus, $1 Y$

Haußmann, Frank, $1 Y$

Heidmann, S., 19

Heininger, $\mathrm{M} ., \mathrm{OH}, \mathrm{OZ}$

Heiter, Ulrike, $2 R$

Hénault, F., OU

Henning, Th., $\mathrm{OH}, \mathrm{OZ}$

Henriquez, N. N., 2L

Henry, Todd J., 2A

Heras, I., 19

Hess, Nicole M., 2E

Hindsley, R. L., 05

Hinz, Phil, OJ, OU, 1M

Hofmann, K.-H., OZ

Hogerheijde, M., OZ

Hönig, Sebastian F., 18, $1 \mathrm{~V}$

Horch, Elliott P., OX, OY, 10, 2A, 2E, 2U

Horton, Anthony, 1J

Hou, Yonghui, 27

Howell, Steve B., 0G, 2E

Hron, J., OZ

Huber, Stefan, 03, ON

Hubin, Norbert, 03, OH, ON

Huby, E., OU, 2V

lacchetta, Alex, $1 \mathrm{~K}$

llee, John, 18

Ireland, Michael J., OU, 11, 18, 1E, 10

Isella, Andrea, 18

Ives, Derek, $\mathrm{OH}, 2 \mathrm{R}$

Jaffe, Walter, $09, \mathrm{OH}, \mathrm{OZ}$

Jakob, G., $\mathrm{OH}$

Jara, R. E., 2L

Jaynes, B., 06

Jeffries, Bryn, $1 \mathrm{~J}$

Jencka, L., 06

Jensen, Eric, 18

Jochum, Lieselotte, $03, \mathrm{OH}$

Jocou, Laurent, 03, 0N, 1Y, 22 
Johnston, Perry, 06, OP

Jolley, Paul, 03

Jones, Jeremy W., 02

Jorgensen, Anders M., 05, 1N

Jovanovic, Nemanja, $1 \mathrm{~J}$

Juanola-Parramon, Roser, 1G, $1 \mathrm{~K}$

Juhasz, Attila, 18

Jung, Yves, $2 R$

Jurgenson, C., 06, OD

Kaiser, Robin, 21

Kammerer, Jens, 1H, 1

Kane, Stephen, 18

Karl, Martina, OK

Keller, C. U., OT

Kellerer, Aglae, IW

Kellner, Stefan, 1 Y

Kelly, Robert, 06, 0M

Kenchington Goldsmith, Harry-Dean, $1 \mathrm{E}$

Kieda, David, OW

Kirchbaver, Jean-Paul, 03, 0N

Kishimoto, Makoto, 18

Kitzmann, Daniel, 11

Klein, Barbara, $2 R$

Klein, R., $\mathrm{OH}$

Klement, Robert, 02

Kley, Wilhelm, 18

Klinglesmith, D., 06

Klupar, Pete, $1 \mathrm{~J}$

Kluska, Jacques, $1 \mathrm{U}$

Kok, Yitping, 1 Y

Kolb, Johann, 03, ON

Kosmalski, Johan, 03, ON

Kral, Quentin, 18

Kratter, Kaitlin, 18

Kraus, Stefan, 0U, 18, 22, 23, 24

Krempl, Peter, 03, ON

Kroes, G., OZ

Labadie, Lucas, 0U, 18, 1B, 1R, 20

Labdon, Aaron, 23

Labeyrie, Guillaume, 21

Lacour, Sylvestre, 07, 0K, 18, 1P, 1W, 1Y, 2V

Lagadec, Tiphaine, OV

Lagarde, Stéphane, 09, 0H, 0Z, 20

Lai, Olivier, 21

Lanthermann, Cyprien, 22, 23, 24

Lapeyrère, Vincent, $1 \mathrm{P}$

Larkin, Kieran, $1 \mathrm{~J}$

LaRue, Ryan, OY

Laughlin, Greg, 18

Laun, W., $\mathrm{OH}, \mathrm{OZ}$

Lawrence, Jon, OV

Lazzarini, Paolo, OF, 2Q

LeBohec, Stephan, OW

Le Bouquin, Jean-Baptiste, 03, ON, 0U, 18, 1P, 22,

23,24

Le Coarer, E., 19, 1S

Léger, A., $1 \mathrm{H}$

Lehmitz, M., $\mathrm{OH}, \mathrm{OZ}$

Leisawitz, David T., $1 \mathrm{~K}$

Le Maître, Matthew, 2F
Lévêque, Samuel, 03

Li, Qian, 27

Ligon, Robert, 06, OD, OM, OP

Lilley, Paul, 03

Lindgren, A., 05

Lippa, Magdalena, OK, $1 Y$

Liu, Y., 29

Lizon, Jean-Louis, 03, 2P, 2R

Lockhart, Matt, 2R

Loicq, J., $1 \mathrm{H}$

Lopez, Bruno, 09, OH, $0 \mathrm{Z}$

Luis, James J. D., 06, OM

Lunde, Emily, 2T

Luther-Davies, Barry, $1 \mathrm{E}$

Ma, Pan, $1 \mathrm{E}$

Macintosh, Bruce, 2G

Madden, Stephen J., $1 \mathrm{E}$

Magnard, Yves, 03, 0N

Magrin, D., 2B

Maher, Stephen F., $1 \mathrm{~K}$

Maier, Erin, OJ, $1 \mathrm{M}$

Majewski, Steven R., $2 U$

Majoinen, Olli, 02

Manescau, Antonio, 2P

Marafatto, L., 2B

Marcotto, A., $\mathrm{OH}$

Mardones, Pedro, 03

Marek, Petr, IW

Marquart, Thomas, $2 R$

Martin, G., OU, 19, 1S, 2V

Martin, P., 2L

Martinache, Frantz, 11

Martinod, Marc-Antoine, 20

Marzotto, Davide, OP

Matter, Alexis, 09, OH, OU, $\mathrm{OZ}$

Matthews, Nolan, OW

Maurer, T., $\mathrm{OH}$

Mauskopf, Philip, 2T

McCracken, T. M., 06, OD

McKeen, C., 06

Megevan, Denis, 2P

Meilland, Antony, 09, OH, $\mathrm{OZ}$

Meisenheimer, $\mathrm{K}$., $\mathrm{OH}, \mathrm{OZ}$

Meister, Alexander, 03, $\mathrm{OH}, \mathrm{ON}$

Mennesson, B., OU

Mentzell, John E., 1G

Mérand, Antoine, 03, 0U, 14, $1 \mathrm{U}$

Meru, Farzana, 18

Mesa, D., 2B

Michael, Ernest A., 18, 1X, 2L, 2M

Michau, Vincent, 20

Millan-Gabet, Rafael, 18

Millour, Florentin, 09, 0H, 0Z, 18

Minardi, Stefano, 0U, 16, 18, 1B, 1R, 20

Molina-Conde, Ignacio, 2R

Monnier, John D., OU, 11, 18, 22, 23, 24

Morand, A., 19

Morbidelli, Alessandro, 18

Mordasini, Chris, 18

Moreau, Vincent, 00 
Morel, Sebastien, 09, OH, $\mathrm{OZ}$

Moreno, E., 2L

Morlok, Andreas, 18

Mortimer, D., 06, 0C

Moulin, Thibaut, $03,0 \mathrm{~N}$

Mourard, Denis, 20

Mozurkewich, Dave, 05, 18, 1N

Mundy, Lee, $1 \mathrm{G}$

Muterspaugh, M., 05

Muthusubramanian, Balaji, IR

Nardetto, Nicolas, 20

Nelson, Richard, 18

Neumann, U., $\mathrm{OH}, \mathrm{OZ}$

Nir, Guy, OQ

Nolte, Stefan, 1B, 1R, 20

Norris, Barnaby R. M., OT, OU, OV, 11, 1E, 1J, 1Q

Norris, Ryan P., $1 T$

Ochoa, D., 06

Ofek, Eran O., OQ

Oliva, Ernesto, $2 R$

Olivares, Andres, 06, 00, OP

Olofsson, Johan, 18

Orban de Xivry, G., OU

Origlia, Livia, $2 R$

Ortega, N., 2L

Osellame, Roberto, 16

Osorio, Juan, 03

Ott, Jürgen, 03

Ott, Thomas, OK, $1 \mathrm{Y}$

Oudmaijer, Rene, 18

Packham, Chris, 18

Paladini, Claudia, 09, 18

Pallanca, Laurent, 03

Panic, Olja, 18

Pantin, E., OZ

Parvex, T., 2L

Pasquini, Luca, 03, 0H, 0N, 2R

Paufique, Jérôme, 03, 2R

Pavez, Marcus, 03

Payne, Ifan, 06, OP, 2F

Pedretti, Ettore, OU, 16, 20

Pepe, Francesco Alfonso, 2P

Percheron, Isabelle, 03

Peronio, Pietro, OY

Perrin, Guy S., 07, OK, 1P, 1Y

Pertsch, Thomas, 20

Peters, Scott, 24

Petit, Cyril, 20

Petrov, Romain G., 09, 0H, 0Z, 18

Pettazzi, Lorenzo, 03

Pfuhl, Oliver, OK, 1 Y

Phan Duc, Thanh, 03

Piacentini, S., 16

Pichon, B., $\mathrm{OH}$

Pilyavsky, Genady, 2T

Piña, M., 2L

Pinna, E., 2B

Pino, Andres, 03

Pino, J., 06

Pirard, Jean Francois, 03
Pirnay, Olivier, 00, OP

Pistunov, Nikolai, 2R

Plattner, Markus, IY

Plewa, Philipp, OK

Pollarolo, C., $2 \mathrm{~L}$

Pooler, S. T., 05

Pope, Benjamin, 18, $1 \mathrm{~J}$

Por, E. H., OT

Portaluri, E., 2B

Pott, Joerg-Uwe, OU, 18, 1R

Poupar, Sébastien, 03

Puglisi, A., 2B

Quanz, Sascha P., 1H, 11

Quentin, Jutta, 03

Quiroga-Nunez, Luis Henry, 18

Ragazzoni, R., 2B

Ramírez, Andres, 03

Ramos Almeida, Cristina, 18

Ramos, N., $2 \mathrm{~L}$

Rates, A., $2 \mathrm{~L}$

Rau, Christian, $1 Y$

Rau, Urvashi, 1C

Raver, H., $1 \mathrm{H}$

Raymond, Sean N., 18

Rearte, C., $2 \mathrm{~L}$

Rech, Ivan, OY

Regaly, Zsolt, 18

Reggiani, M., OU

Reiners, Ansgar, 2R

Restaino, Sergio R., 05, OB, 2 I

Reyes, Javier, 03, 0N

Reynolds, Mark, 18

Ridgway, Stephen T., 02, 18

Ridings, Robert, 03

Rinehart, Stephen A., 18, 1G, $1 \mathrm{~K}$

Riquelme, M., $\mathrm{OH}$

Riva, Marco, $2 \mathrm{P}$

Rivet, Jean Pierre, 21

Rivinius, Thomas, 03, 09

Rivinus, Th., $\mathrm{OH}$

Rizzo, Maxime J., $1 \mathrm{G}$

Robbe-Dubois, Sylvie, 09, OH, $0 \mathrm{Z}$

Rochat, Sylvain, 03, ON

Rodriguez, A., 2L

Romero, V. D., 06

Rousseau, Sylvain, 09, 0H, 2Q

Rousselet-Perraut, Karine, 07, 0K, 1P, 1Y, 20

Ruffio, Jean-Baptiste, 2G

Rupert, Justin D., OX, OY

Sakurai, T., 29

Salcido, Christopher, 06, OD, OP

Salgado, Fernando, 03

Sallum, Steph, 1D

Samain, Etienne, 21

San Martin, C., $2 L$

Sánchez-Bermudez, Joel, $1 U$

Santoro, Fernando, 06, OD, OP

Satoor, Tanish, $2 \mathrm{~F}$

Savransky, Dmitry, 2G

Schaefer, Gail H., 02 
Schertl, D., OZ

Schifano, L., $1 \mathrm{H}$

Schmidt, L. M., 06, OD

Schmitt, Henrique R., 05, OB, 2D, 2 I

Schödel, Rainer, $1 \mathrm{U}$

Schoeller, Markus, 03, 09, OH, OK, OZ

Schreiber, Matthias, 18

Schuhler, Nicolas, $03, \mathrm{OH}, \mathrm{ON}$

Scott, Nicholas J., 0G, 12

Seemann, UIf, $2 R$

Seneta, E. B., 06

Sepulveda, S., $2 \mathrm{~L}$

Serabyn, E., OU

Setterholm, Benjamin R., 22, 23, 24

Shankar Nayak, Abani, 20

Shankland, P., 05

Shao, Mike, 1J

Shapiro, Jacob, 2G

Shchekaturov, Pavel, 03, 0N

Sinclair, Adrian, $2 \mathrm{~T}$

Skemer, Andy, 1D

Smette, Alain, 2R

Smith, Michael, 18

Smoker, Jonathan, 2R

Snik, F., OT

Snow, Shaun, OW

Song, T.F., 29

Soulain, A., $\mathrm{OH}$

Soulez, Ferréol, 10

Spalding, Eckhart, 0J

Spang, A., $\mathrm{OH}$

Stadler, Eric, ON

Stangalini, M., 2B

Stassun, Keivan, 18

Stee, Ph., $\mathrm{OH}, \mathrm{OZ}$

Stempels, Eric, $2 R$

Stephan, Christian, $03, \mathrm{OH}, \mathrm{ON}$

Stone, Jordan, 0J

Straubmeier, Christian, 07, 0K, IP, IY

Sturm, Eckhard, IY

Sturmann, Judit, 02

Sturmann, Laszlo, 02

Suarez, Marcos, 03

Sun, X., 06

Surdej, Jean, OU, 18

Tallon, Michel, 1U, 20

Tallon-Bosc, Isabelle, 1U, 20

Tamblay, Richard, 03

Tapia, Mario, 03

ten Brummelaar, Theo A., 02, 18, 20, 22, 23, 24

Tepper, Jan, 1B, 1R, 20

Thayer, Patrick R., 2E

Thiébaut, Éric, $1 \mathrm{U}$

Tian, F., $1 \mathrm{H}$

Toledo, P., $\mathrm{OH}$

Tristam, $\mathrm{K}$., $\mathrm{OH}$

Tristram, Konrad R. W., 03, 0U, 18, 1V

Turner, Neal, 18

Turner, Nils H., 02

Tuthill, Peter, OS, OT, OV, 11, 18, 1E, $1 \mathrm{~J}$
Ulliac, G., 19, 2V

Umbriaco, G., 2B

Vakili, Farrokh, 21

Valdes, Guillermo, 03, 0N

Valenti, Elena, $2 R$

van Belle, Gerard T., 05, OB, 18, 2A

van Boekel, R., $\mathrm{OZ}$

van der Heyden, Pierre, 03

Varga, Jozsef, 09, OH

Vargas, Norman L., 02

Vasisht, Gautum, 18

Vassallo, D., 2B

Veach, Todd, $1 G$

Veillet, Christian, 28

Vérinaud, Christophe, 03, 0N

Vernet, David, 21

Vila Hernandez de Lorenzo, Jordi, IG

Viotto, V., 2B

Waisberg, Idel, OK

Wallace, Alexander, 18

Wang, Rongping, $1 \mathrm{E}$

Wang, Yanqiang, 27

Webster, Larry, 02

Weeks, Eric, 2T

Weigelt, Gerd, $\mathrm{OH}, 0 \mathrm{OZ}, 18$

Weiss, Samuel A., OX, OY

Wheeler, Caleb, $2 \mathrm{~T}$

Widmann, Felix, OK

Wieprecht, Ekkehard, IY

Wiezorrek, Erich, $1 Y$

Wilby, M. J., OT

Wilson, Donald M. A., 06, 26

Winters, Jennifer G., 2A

Wishnow, Edward, 18

Withford, Michael J., OV, 1B, 1R, 1S

Wittkowski, Markus, 18

Woillez, Julien, 03, OH, 0N, OU, 11

Wolf, Sebastian, $0 \mathrm{Z}, 18$

Wolff, Burkhard, IP

Woods, Craig, 02

Wu, Zhen, 27

XU, Mingming, 27

$\mathrm{Xu}$, Teng, 27

Young, John, 06, 0D, OP, 18, 10, 1U, 2F

Zackay, Barak, $0 Q$

Zavala, R. T., 05, 2D

Zhang, Huatao, 27

Zhang, X.F., 29

Zhao, M.Y., 29

Zhao, Ming, 18

Zhu, Zhaohuan, 18

Zins, Gérard, 03, 09, OH, $0 \mathrm{Z}$

Zúñiga-Fernández, Sebastian, 18 
Proc. of SPIE Vol. 10701 1070101-16

Downloaded From: https://www.spiedigitallibrary.org/conference-proceedings-of-spie on 26 Apr 2023 Terms of Use: https://www.spiedigitallibrary.org/terms-of-use 


\section{Conference Committee}

Symposium Chairs

Allison A. Barto, Ball Aerospace \& Technologies Corporation (United States)

Suzanne K. Ramsay, European Southern Observatory (Germany)

Symposium Co-chairs

Satoru Iguchi, National Astronomical Observatory of Japan (Japan)

Alison B. Peck, Gemini Observatory (United States)

Conference Chairs

Michelle J. Creech-Eakman, New Mexico Institute of Mining and Technology (United States)

Peter G. Tuthill, The University of Sydney (Australia)

Antoine Mérand, European Southern Observatory (Chile)

\section{Conference Program Committee}

Ellyn K. Baines, U.S. Naval Research Laboratory (United States)

Fabien Baron, Georgia State University (United States)

Jean-Philippe Berger, European Southern Observatory (Chile)

Elliott P. Horch, Southern Connecticut State University (United States)

Takayuki Kotani, National Astronomical Observatory of Japan (Japan)

Lucas Labadie, Universität zu Köln (Germany)

Fabien Malbet, Institut de Planétologie et d'Astrophysique de Grenoble (France)

Keiichi Ohnaka, Universidad Católica del Norte (Chile)

Claudia Paladini, Université Libre de Bruxelles (Belgium)

Stephanie Sallum, University of California, Santa Cruz (United States)

Isabelle Tallon-Bosc, Centre de Recherche Astronomique de Lyon

(France)

Session Chairs

1 Current and Planned Facilities I

Antoine Mérand, European Southern Observatory (Chile)

2 Current and Planned Facilities II

Peter G. Tuthill, The University of Sydney (Australia) 
3 Current and Planned Facilities III

Michelle J. Creech-Eakman, New Mexico Institute of Mining and Technology (United States)

4 Techniques I

Stephanie Sallum, University of California, Santa Cruz (United States)

5 Techniques II

Ellyn K. Baines, U.S. Naval Research Laboratory (United States)

6 Techniques III

Michelle J. Creech-Eakman, New Mexico Institute of Mining and Technology (United States)

$7 \quad$ Future of Interferometry I

Michelle J. Creech-Eakman, New Mexico Institute of Mining and Technology (United States)

8 Future of Interferometry II

Jean-Philippe Berger, European Southern Observatory (Chile)

9 Data Processing I

Claudia Paladini, Université Libre de Bruxelles (Belgium)

10 Space Interferometry

Elliott P. Horch, Southern Connecticut State University (United States)

11 Data Processing II

Fabien Baron, Georgia State University (United States)

12 Technologies I

Takayuki Kotani, National Astronomical Observatory of Japan (Japan)

13 Technologies II

Lucas Labadie, Universität zu Köln (Germany)

14 Critical Subsystems

Antoine Mérand, European Southern Observatory (Chile)

15 Michelson, Fizeau and Thesis Prizes

Michelle J. Creech-Eakman, New Mexico Institute of Mining and Technology (United States)

Peter G. Tuthill, The University of Sydney (Australia)

16 Critical Subsystems II

Peter G. Tuthill, The University of Sydney (Australia) 


\section{Introduction}

The 2018 Optical and Infrared Interferometry and Imaging VI conference included many new developments in the astronomical interferometry field, which has expanded this year with particular growth in the disciplines of speckle, aperture masking and intensity interferometry. These areas are experiencing a resurgence due to their utility on large aperture telescopes, as well as the availability of highspeed, low noise detectors and advanced computer systems. We are also learning of more developments for balloon-borne interferometry and potential interferometric approaches on future space platforms. Finally, this conference saw exciting new avenues of inquiry in nulling techniques, adaptive optics, photonics, improved fiber technologies, photon-counting detectors and new beam combiners using novel approaches and combinations of multiple technological advances only recently available.

Highlights of this conference include updates from all the large facilities operating and under development today: CHARA, LBTI, MROI, NPOI and VLTI. We heard from the GRAVITY team on the results of microarcsecond measurements of the galactic center. New instruments or facilities are coming online or in various stages of development, including the Planet Formation Imager (PFI), MATISSE, Hi-5, SPICA, MYSTIC, MIRC-X and FOURIER. In the speckle community recent instruments and scientific results were presented, including from 'Alopeke and NESSI, while Cherenkov arrays and new approaches to intensity interferometry with small aperture telescopes showed new promise in this venerable field. Science highlights ranged from discussions of young stellar objects and active galactic nuclei, to stellar convection/spots and exoplanets. The community continues to actively work from optical through thermal infrared, with many synergistic approaches across wavelengths as well as science inquiries utilizing more traditional astronomical techniques to fully constrain the investigations. High angular resolution continues to produce unique insights for the astronomical community that cannot be garnered in any other ways.

We resumed community awards at this meeting. From the strongly competitive field of five dissertation papers, the committee chose to award the best dissertation presentation to Tiphaine Lagadec, The Univ. of Sydney (Australia) for her work on the GLINT South project on the AAT. The Michelson Lifetime Achievement award for his work at Georgia State Univ. (United States) on speckle and long-baseline interferometry through CHARA went to Dr. Hal McAlister. The Fizeau Lifetime Achievement award for his work at Harvard-CfA and NASA-JPL including crucial roles on IOTA, SIM, and the Keck and LBT Interferometers went to Dr. Wesley Traub. The Michelson Investigator award for his work on Cepheids, evolved stars and fundamental stellar parameters went to Dr. Pierre Kervella. The Michelson Awards, previously supported by the Mount Wilson Observatory (United States), are now being sponsored by Lowell Observatory (United States); the Fizeau Awards 
continue to be supported by l'Observatoire de la Côte d'Azur (France). Finally, the imaging contest (we have moved past the "beauty" moniker) also contained many interesting approaches to the modeling of our "secret target" and the interested reader will have to seek out the paper led by Antoine Mérand, European Southern Observatory (Germany) in these proceedings to learn who won the competition and why.

We invite you to peruse this volume from SPIE to learn of the latest developments in our fast-paced and innovative field.

Michelle J. Creech-Eakman

Peter G. Tuthill Antoine Mérand 